\title{
A comprehensive computational mutation structure- function approach for determining potential drug target sites in poliovirus 2A protease
}

\author{
Amna Younus*, Muhammad Faraz Bhatti \\ Atta-ur-Rahman School of Applied Biosciences, National University of Sciences and Technology, Sector H-12, Kashmir \\ Highway, Islamabad 44000, Pakistan \\ *For correspondence: Email: amnayounus82@yahoo.com, Tel: 0092-305-5869935; Fax: 0092-51-90856102 \\ Sent for review: 5 September 2017 \\ Revised accepted: 16 November 2017
}

\begin{abstract}
Purpose: To investigate a computational approach for analysing the structure-function relationship of poliovirus $2 A$ protease using various bioinformatics tools.

Methods: The three-dimensional structure of 2Apro was modelled and analyzed using the crystal structure of coxsakievirus B4 as a template to understand the function of this protein. Structural validation programs, VADAR and QMEAN, were used to verify the 2Apro model. Analysis of protein stability changes in poliovirus $2 A$ protease-mutated sequences using various servers was also performed. Furthermore, mutation pattern, intrinsic disorder regions (IDRs), hydrophobic regions, drug binding sites (DBS) and subcellular localization were identified.

Results: Hydrophobicity results confirmed the suitability and reliability of $2 A$ protease as a potential drug target. Less IDRs were observed in the protein. Moreover, the results showed the presence of various important drug binding targets among conserved regions of the protease. The predicted drug binding sites indicate their suitability for the inhibition and development of anti-viral drugs against poliovirus $2 A$ protease.

Conclusion: The current study resulted in the detection of important ligand interactions with respect to the binding site of the targeted protein. Thus, these compounds may be potent drug candidates and their potency may be increased against poliovirus $2 A$ protease with relatively simple structural changes.
\end{abstract}

Keywords: 2A Protease, Computational analysis, Drug binding sites, Intrinsic disorder regions, Hydrophobicity

\begin{abstract}
This is an Open Access article that uses a funding model which does not charge readers or their institutions for access and distributed under the terms of the Creative Commons Attribution License (http://creativecommons.org/licenses/by/4.0) and the Budapest Open Access Initiative (http://www.budapestopenaccessinitiative.org/read), which permit unrestricted use, distribution, and reproduction in any medium, provided the original work is properly credited.
\end{abstract}

Tropical Journal of Pharmaceutical Research is indexed by Science Citation Index (SciSearch), Scopus, International Pharmaceutical Abstract, Chemical Abstracts, Embase, Index Copernicus, EBSCO, African Index Medicus, JournalSeek, Journal Citation Reports/Science Edition, Directory of Open Access Journals (DOAJ), African Journal Online, Bioline International, Open-J-Gate and Pharmacy Abstracts

\section{INTRODUCTION}

Viral proteins involved in the cellular functions and manipulation of cellular processes by viruses have gained significant attention from the pharmaceutical and biotechnology industries as a potential source of drug targets [1]. Poliovirus proteinases are excellent substrates for drug development due to their key roles in the replication cycle.

Drug targets are proteins or protein products 
including G-protein coupled receptors, enzymes, ion channels and amino acids. To accomplish desirable therapeutic effects drugs tend to bind to their matching targets [2]. The extreme genetic variability and high mutation rate of RNA viruses such as poliovirus makes the design of antiviral drugs complicated. Polioviruses are therefore termed "quasi-species" [3-5].

Poliovirus (PV) of the Picornaviridae, has been categorized into three stable subtypes (1,2 and $3)$. The PV genome comprises +ss RNA of approximately 7,440 nucleotides. The genome is packaged in a non-enveloped icosahedral protein capsid [6]. PV replication is carried out through a single open reading frame termed a polyprotein. Two cysteine proteases (2A and $3 \mathrm{C}$ ) are involved in proteolytic processing of polyprotein, which are crucial for viral life cycle [7]. The poliomyelitis epidemic resulted in the discovery of two important vaccines, OPV and IPV. No drug is available for treating poliomyelitis.

PV $2 A^{\text {pro }}$ plays a leading role in autocatalytic cleavage process of poliovirus polyprotein. $2 A^{\text {pro }}$ is involved in cleavage of elF4G (eukaryotic translation initiation factor $4 \mathrm{G}$ [8]. Studies have demonstrated the role of poliovirus $2 A^{\text {pro }}$ in activating the apoptotic process in PV-infected cells, and it also exhibits anti-apoptotic activity [9].

The poliovirus $2 \mathrm{~A}^{\text {pro }}$ crystal structure is unknown; however, it is possibly very close to the known structure of HRV2 $2 A^{\text {pro }}[10]$. Research on $2 A^{\text {pro }}$ inhibitors has been limited so far. However, a number of peptide-based inhibitors have been developed against $2 A^{\text {pro }}$. Anti-2A inhibitors are expected to show the additional advantage of suppressing genetic reversion along with negative effect son capsid formation [11]. Poliovirus proteases display minimal similarity to known mammalian enzymes, making them excellent drug targets [12]. The aim of this study was to explore poliovirus $2 \mathrm{~A}$ protease as a probable drug target through computational approaches. Sequences in which mutations were already reported were used [13]. Analysis was performed to investigate drug target sites of 2Apro. We also investigated the effect of mutations on protein stability. The degree of conservation was mapped on the 3D structure of the proteins and compared with the novel predicted intrinsic disordered regions (IDRs) of poliovirus 2A protease (Figure 1).

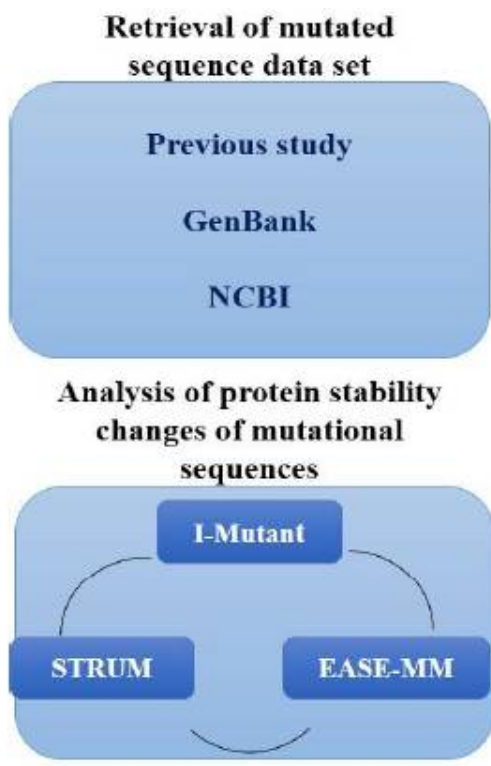

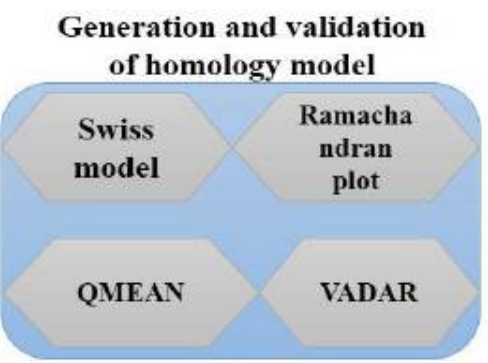

Prediction of intrinsic disorder regions (IDRs)

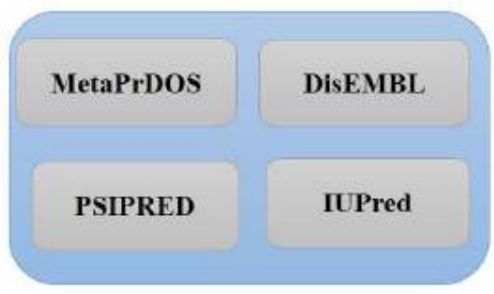

Prediction of 3D structure

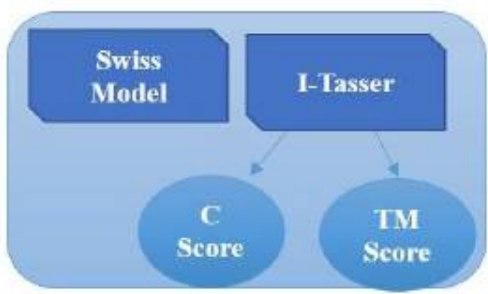

Prediction of hydrophobicity

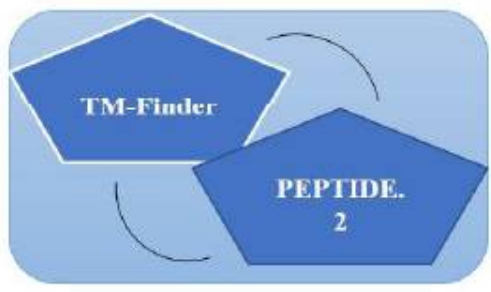

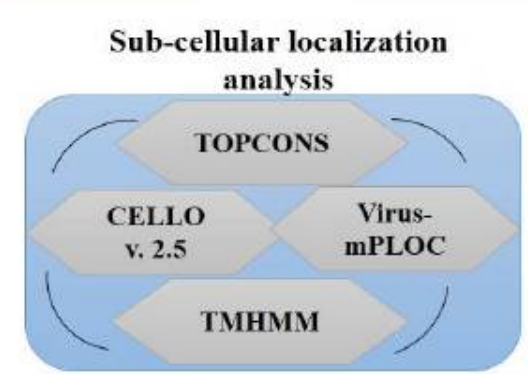

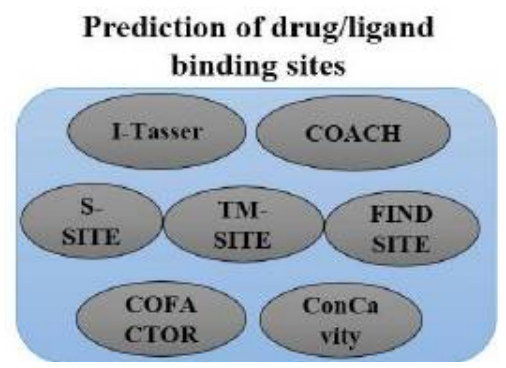

Figure 1: Schematic representation of prioritization pipeline in identifying regions with more DBS and least IDS based on various in-silico tools 


\section{EXPERIMENTAL}

\section{Sequence retrieval}

The 2A protease was amplified from blood samples (001 and 002) (from polio patients in the KP district of Pakistan with the help of Frontier Development Organization) then cloned into a TA cloning vector (PCR 2.1) and sent for sequencing (Eurofin, USA). The sequences were analyzed and submitted to Genbank (KF193869 and KF193870 for 001 and 002 respectively) [13]. Total of four mutations in the poliovirus $2 A$ protease were observed after sequencing. Alanine residue (at position 12) was mutated into glycine, while wild type aspartic acid residue at position 36 was mutated into asparagine and normal serine was mutated to threonine at position 134 and isoleucine mutated to valine at amino acid position 136.

\section{Homology modelling and model reputation}

A PDB file of 001 and 002 samples of $2 A$ protease was generated by the Swiss-Model server [14]. The structure of coxsackievirus B4 (1z8r) model was selected as a template to obtain the $2 \mathrm{~A}$ model. The backbone conformation of the modelled structure of 001 and 002 proteases was calculated by investigating the torsion angles via Ramachandran plot version 2.0 [15]. Further analysis of the predicted model was done by QMEAN [16] and ProSA [17]. VADAR was performed to determine the volume area dihedral angle for fractional accessible surface area [18].

\section{Protein structure prediction}

I-Tasser [19] server was used to predict protein structure by giving precise structural and functional predictions by means of algorithms [20]. A total of five models were obtained based on the five largest structure clusters. Confidence scores (C-score) were calculated based on the significance of threading template alignments. Cscores lied in the range of $[-5,2]$, where a higher value indicates a model with a high confidence and vice-versa. A TM-score (template modelling score) of $>0.5$ predicts a model of correct topology while a TM-score $<0.17$ specifies a random similarity. $2 A^{\text {pro }}$ sequences containing mutations were subjected to structure prediction by I-Tasser.

\section{Protein stability prediction}

I-Mutant 2.0 [21] network predicts protein stability changes upon single point mutation from protein sequence/structure [22]. Output is obtained in the form of protein stability change and Gibbs-free energy change (DDG) using Eq 1.

$\mathrm{DDG}=\mathrm{DG}$ (mutant Protein) $-\mathrm{DG}$ (wild-type, $\mathrm{kcal} / \mathrm{mol}) \ldots . . .(1)$

Furthermore, STRUM was used to predict fold stability change $(\Delta \Delta \mathrm{G})$ of protein molecules upon single-point mutations [23].

EASE-MM sequence-based prediction of mutation-induced stability changes [24], was also used to affirm the stability of the protein upon mutations. Results are displayed in the form of an increase or decrease in stability upon mutation induction.

Protein intrinsic disorder region (IDR) analysis

Variations in IDR structural properties can play a regulatory role in protein activity. The IDRs of the 001 and 002 poliovirus 2A protease, were estimated using four software packages: MetaPrDOS [25], DisEMBL [26], PSIPRED [27] and IUPred [28]. A disorder score (0-1) for each amino acid position was calculated. Position of an amino acid was considered IDR if its disorder score was observed above the cut-off value of 0.5 .

\section{Prediction of hydrophobicity}

Prediction of overall hydrophobic residues of 001 and 002 poliovirus $2 \mathrm{~A}$ protease was performed using the TM Finder tool [29]. The TM-Finder predicts trans-membrane proteins. The regions are predicted to be trans-membrane based on the hydrophobicity and helicity of the adjacent amino acid sequences. The threshold value for hydrophobicity is 0.4 and values exceeding the threshold level predict the transmembrane nature of the protein [29]. Hydrophobicity results of the protein were also confirmed using the PEPTIDE.2 server.

\section{Prediction protein-ligand-drug binding site}

Tertiary structures of 001 and 002 poliovirus 2A protease were analyzed to predict the number of drug binding sites. The online servers $\mathrm{COACH}$ $[19,30]$ I-Tasser were used to determine the number of binding sites and their positions. $\mathrm{COACH}$ is a meta-server approach for the prediction for ligand binding targets through two comparative methods TM-SITE [31] and S-SITE. The BioLiP protein function database is used in these methods to recognize ligand binding sites. These predictions were later combined with the results obtained from other methods including ConCavity [32], FINDSITE [33] and COFACTOR 
[20]. To predict the final ligand binding site of the protein, PDB structures of 001 and 002 poliovirus $2 A$ protease were given to the server for analysis. Complete results were given in the form of the position of the potential drug binding site.

\section{Subcellular localization analysis}

To understand a protein's function and its suitability as a vaccine/target, subcellular localization analysis is performed. Cytoplasmic proteins tend to have more potential as drug targets compared to surface membrane proteins [34]. The CELLO v.2.5 [35] server was employed to predict subcellular localization of poliovirus $2 \mathrm{~A}$ protease. The results were further validated with predictions obtained from Virus-mPLoc [36], TOPCONS [37] and TMHMM [38].

\section{RESULTS}

\section{Structures of 001 and 002 PV2A protease models}

The Swiss-Model predicts the 3D structure of both the models on the basis of known crystal structure of the protein (Figure 2). The maximum scoring and validated model for PV2A protease exhibiting the highest sequence identity with the crystal structure was coxsackievirus B4 (PDB ID: 1Z8R.A).

The stereo-chemical attributes of the predicted protein models were investigated through QMEAN (Table 1) and evaluated by Ramachandran plots (Figure 3).

The QMEAN4 and QMEAN6 score [39] of the model was close to the value of 0 , showing the reliability of the model (model reliability in between 0 and 1; Table 1). Predicted structure analysis delivered solid evidence of the good quality of predicted 3D structure of poliovirus $2 \mathrm{~A}$ protease.

Protein model quality and accessible surface area (ASA) was predicted through VADAR. Accessible surface area to water molecules was measured as fractional ASA range (0 to 1). Results of ASA showed that the major portion of the protein is hydrophobic, having ASA scores less than 0.8 signifying tight folding of the protein (Figure 4).

\section{Predicted drug binding sites (DBS)}

DBS analysis predicted functional ligand binding sites. All of these binding site residues of both models were also confirmed when predicted through different methods including COFACTOR, FINDSITE, TM-SITE and ConCavity. In the case of COFACTOR His 4, Gln 5, Asn 6, Lys 7, Ala 8, His 20, Leu 21, Asp 38, Cys 55, Cys 57, Gln 81, Tyr 82, Met 83, Glu 84, Asn 86, Tyr 88, Tyr 89, Arg 92, Gln 94, Asp 108, Cys 115, His 117, Ile 123, Thr 124, Ala 125, Gly 126, Gly 127, Leu 130 and Ala 132 were predicted as potential binding sites for 001 and 002 (Figure 5A and 5B). In contrast, FINDSITE predicted Phe 2, Gly 3, His 4, Gln 5, Asn 6, Lys 7, His 20, Leu 21, Ala 22, Thr 24, Glu 25, Asp 26, Leu 27, Ser 29, Val 30, Asn 31, Val 33, Asp 38, Val 41, Thr 42, Arg 45, Gln 47, Ser 51, Lys 69, Tyr 83, Asn 86, Tyr 89, Ala 91, Arg 92, Gly 107, Asp 108, Cys 109, Ile 123, Thr 124, Ala 125, Gly 126, Gly 127 , Gly 129 , Val 131 and Phe 133 as potential binding sites (Figure 5C and 5D).

His 20, Leu 21, Asp 38, Cys 55, Cys 57, Met 83, Glu 84, Arg 92, Gln 94, Asp 108, Cys 115, His 117, Ile 123, Thr 124, Ala 125 and Gly 126 were predicted as potential drug binding sites of 001 and 002 according to TM-SITE results (Figure 6A and $6 \mathrm{~B}$ ), while ConCavity results predicted Gln 5 , Asn 6, Lys 7, Ala 8, Asn 18, Tyr 19, His 20, Leu 21, Asp 38, Asp 50, Tyr 62, Tyr 71, Tyr 83, Asn 87, Tyr 88, Tyr 89, Gln 94, Gly 100 , Gly 102 , Phe 103, Ala 104, Pro 106, Gly 107, Cys 109, Gly 110, Ile 112, Arg 114, Ile 123, Thr 124, Gly 126, Gly 129 and Val 131 as potential drug binding residues (Figure 6C and 6D).

\section{Intrinsic disorder region prediction}

To understand protein folding and function, precise prediction of a protein's tendency to have intrinsic disordered regions is an essential step. The current investigation used four software packages to identify disordered positions based on the majority voting by these software tools. In this prediction server, a residue is considered as disordered if its score is above 0.5 . Based on the real valued disorder propensities generated by MetaPrDOS [25], DisEMBL [26], PSIPRED [27] and IUPred [28] analysis, disordered regions are defined as regions with scores above the threshold line (0.5); however, scores below this line specify a degree of flexibility. In the case of 001 and 002, regions GFGH (1-4) and EAMEQ (145-149) were found in ID regions.

\section{Subcellular localization}

Subcellular localization prediction of PV2A protease specifies its localization in the cytoplasmic membrane with no signal peptide and transmembrane helix. 

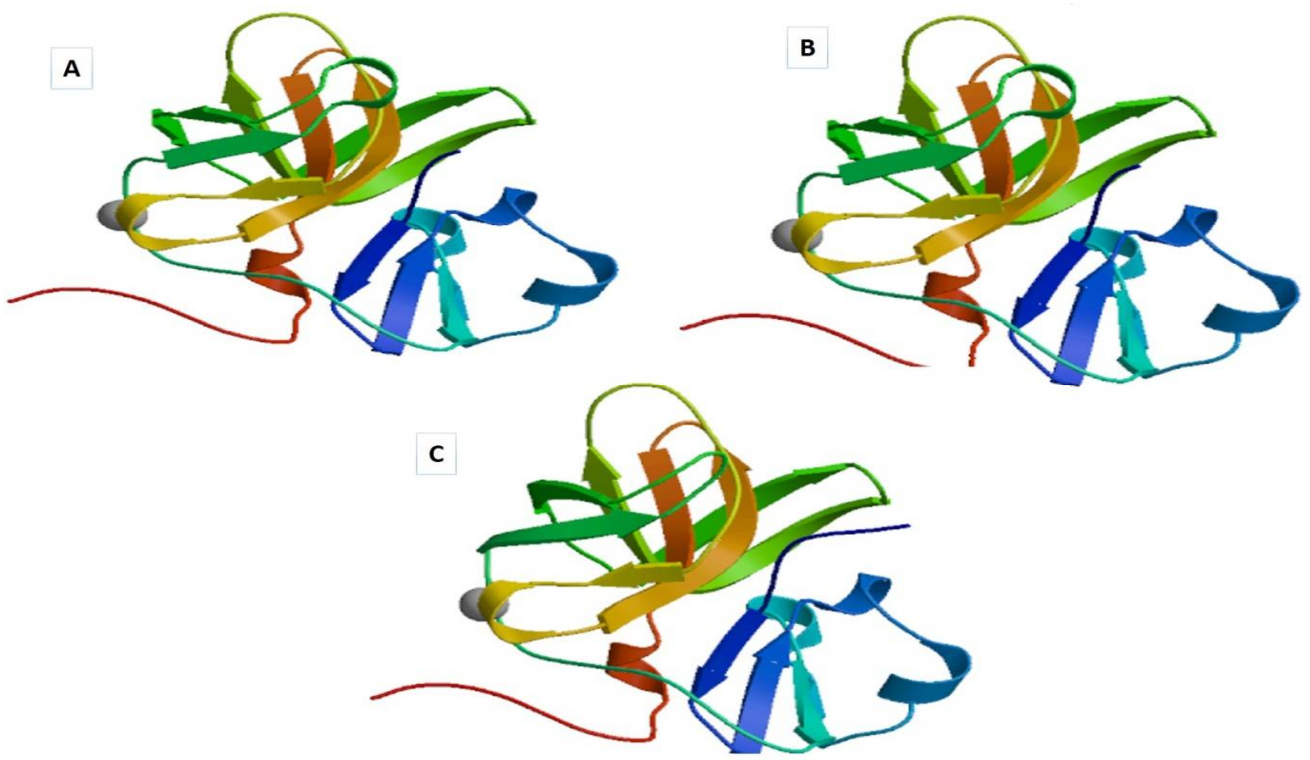

Figure 2: $3 D$ model generation through Swiss model software. $A$ and $B$ indicate the model produced for poliovirus $2 \mathrm{~A}$ protease for sample 001 and 002 respectively. Whereas, $C$ is the template model (1z8r) used for analysis
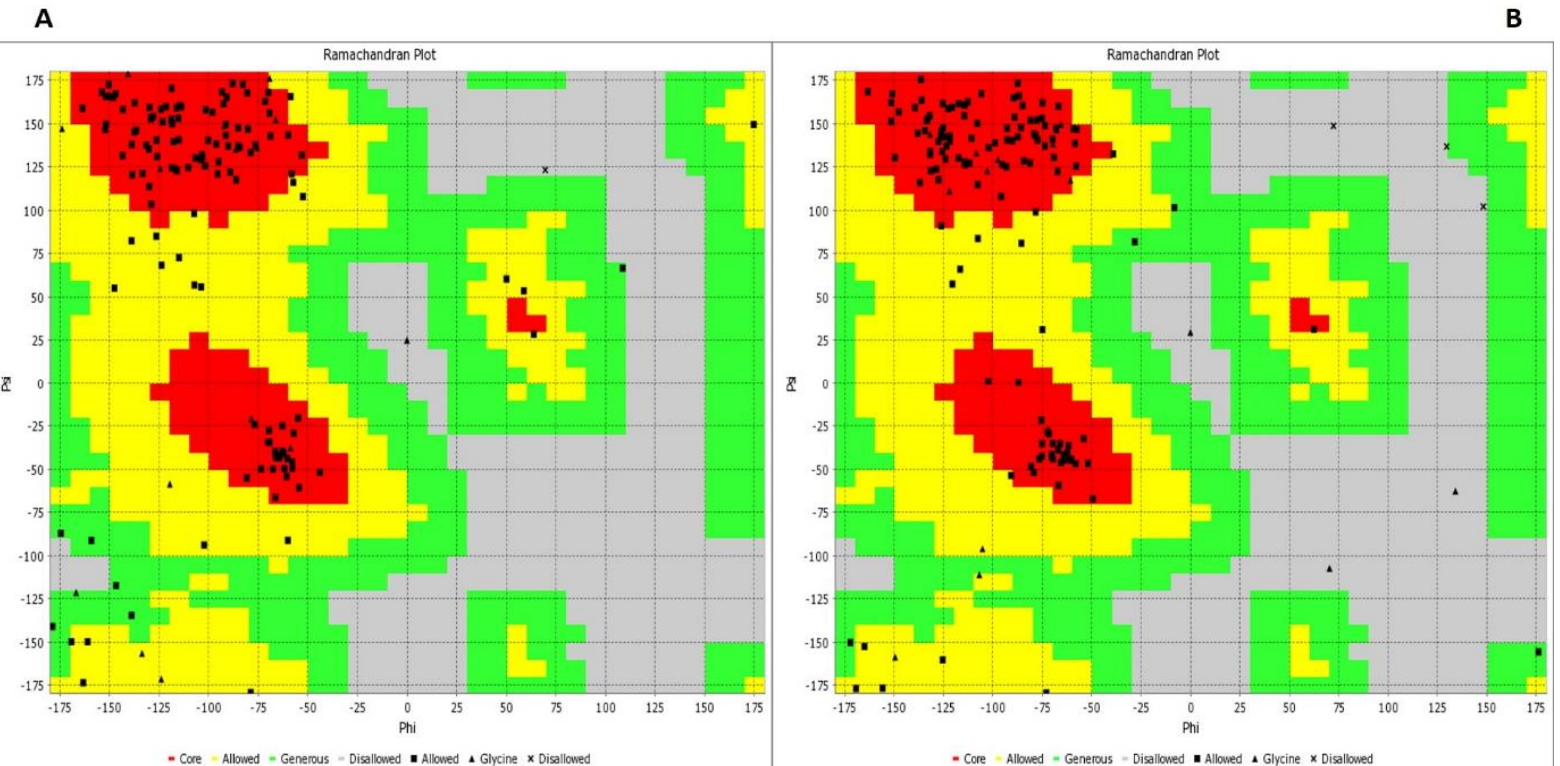

Figure 3: Ramachandran plot analysis of 001 (A) and 002 (B) protein. Red colour indicates core region, yellow indicates allowed region, green is allowed region, grey is disallowed region

Table 1: QMEAN global scores for 001 002, and template models obtained from Swiss modelling

\begin{tabular}{|c|c|c|c|}
\hline \multirow{2}{*}{ Scoring function term } & \multicolumn{3}{|c|}{$\begin{array}{c}\text { Z-score } \\
\text { (Reliability range } 0-1 \text { ) }\end{array}$} \\
\hline & 001 & 002 & $\begin{array}{c}\text { Template } \\
1 Z 8 R\end{array}$ \\
\hline QMEAN6 score & -4.16 & -3.55 & -4.18 \\
\hline QMEAN4 score & -4.12 & -3.41 & -3.92 \\
\hline All-atom pairwise energy & -2.23 & -2.28 & -2.48 \\
\hline C_beta interaction energy & -0.71 & -0.84 & -1.43 \\
\hline Solvation energy & -1.51 & -1.56 & -1.91 \\
\hline Torsion angle energy & -3.57 & -2.82 & -3.17 \\
\hline SS Agreement & -1.38 & -1.04 & -1.34 \\
\hline ACC Agreement & -0.91 & -1.24 & -1.31 \\
\hline
\end{tabular}


A

Fractional Accessible Surface Area

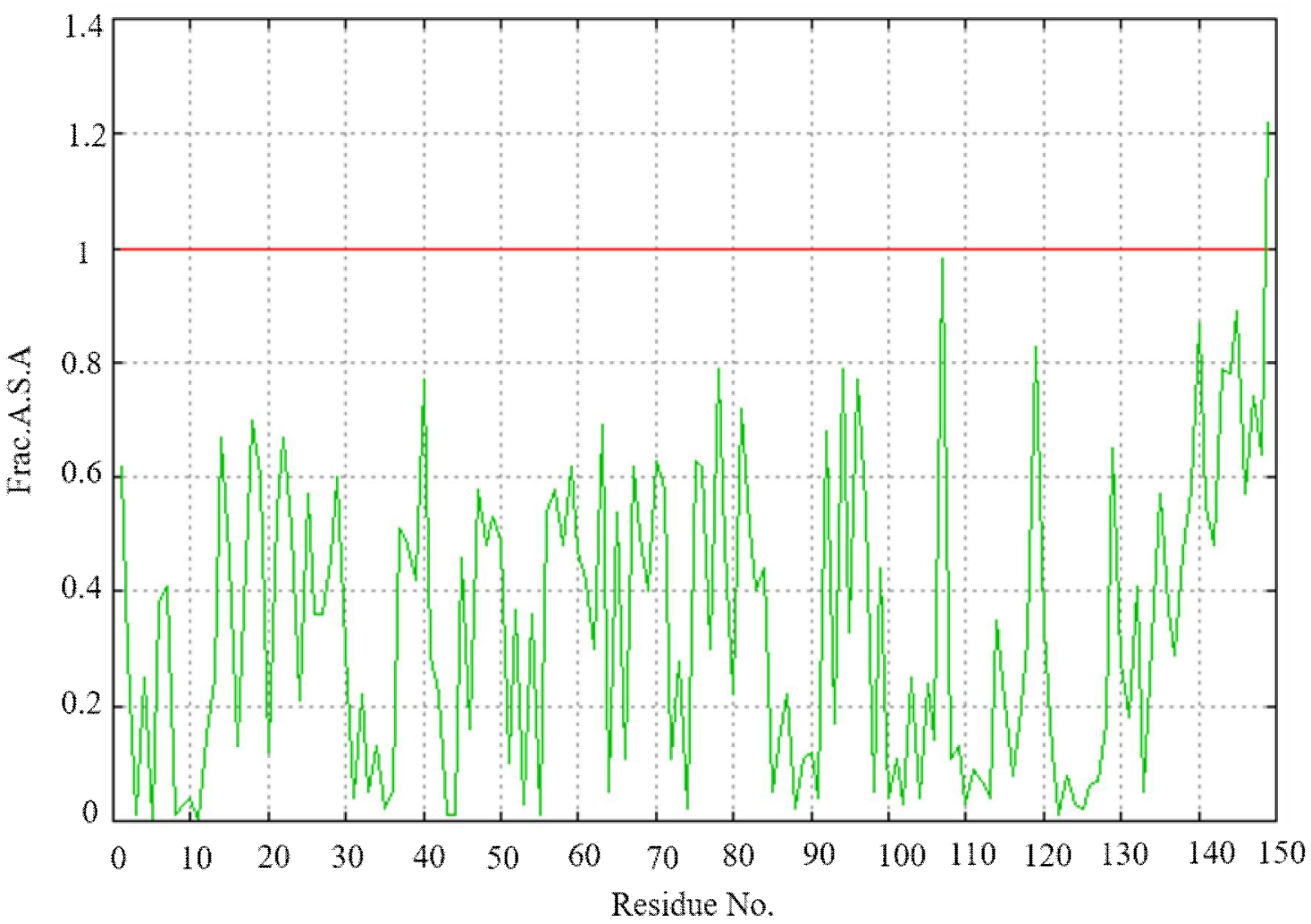

$\mathbf{B}$

Fractional Accessible Surface Area

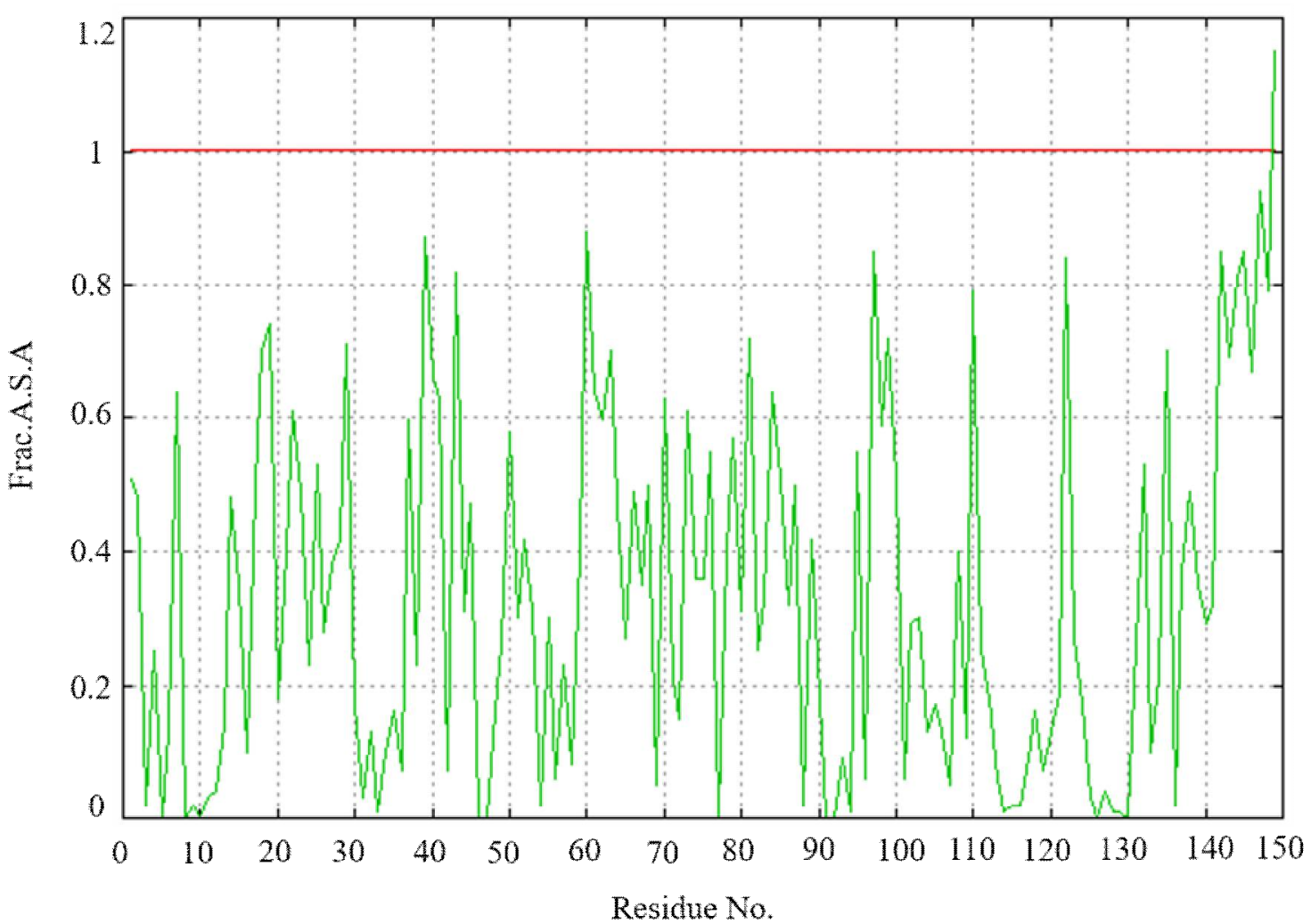

Figure 4: Accessible surface area (ASA) prediction of $001(\mathrm{~A})$ and $002(\mathrm{~B})$ protease models using VADAR server 


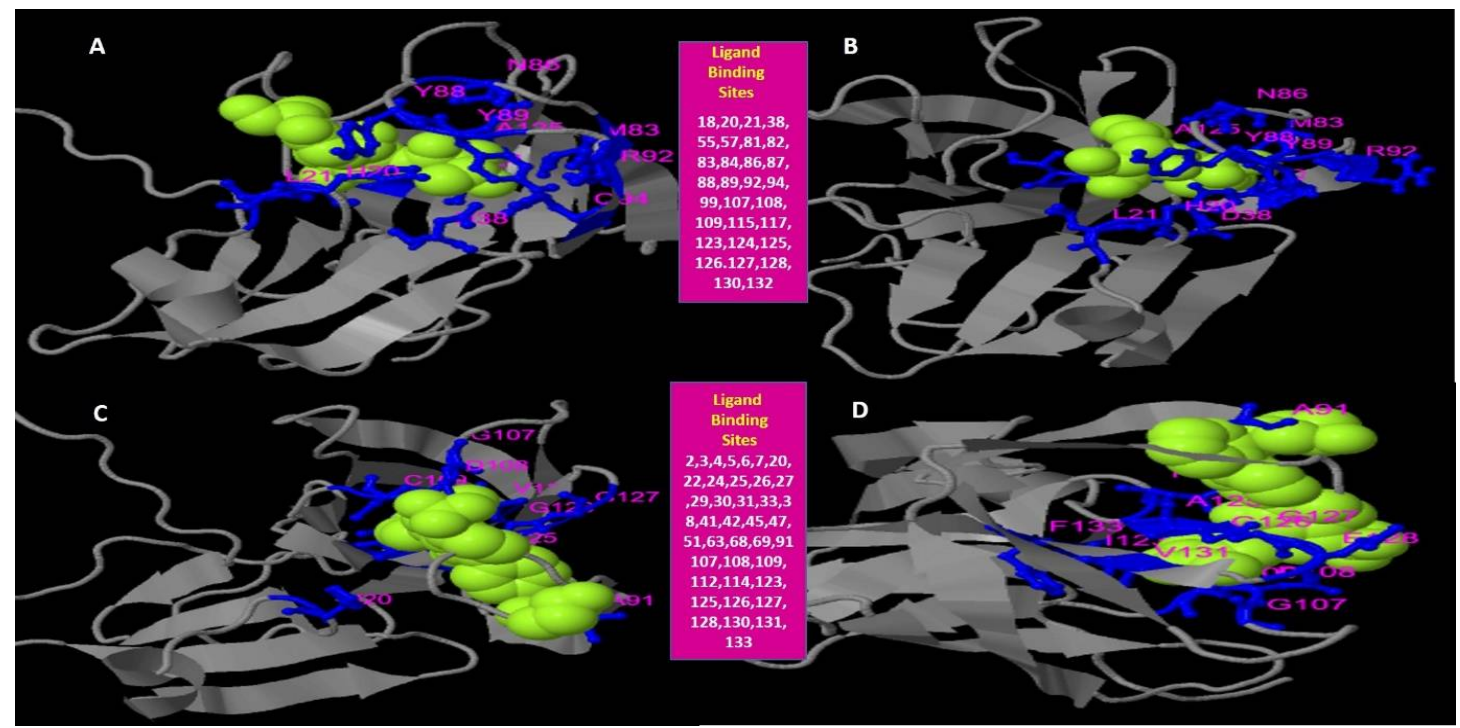

Figure 5: Ligand binding sites prediction through $\mathrm{COACH}$ server. $\mathrm{A}$ and $\mathrm{B}$ are the predictions from COFACTOR of 001 and 002 respectively, while $C$ and $D$ are the results of FINDSITE methods. Ligand binding sites are shown in purple colour

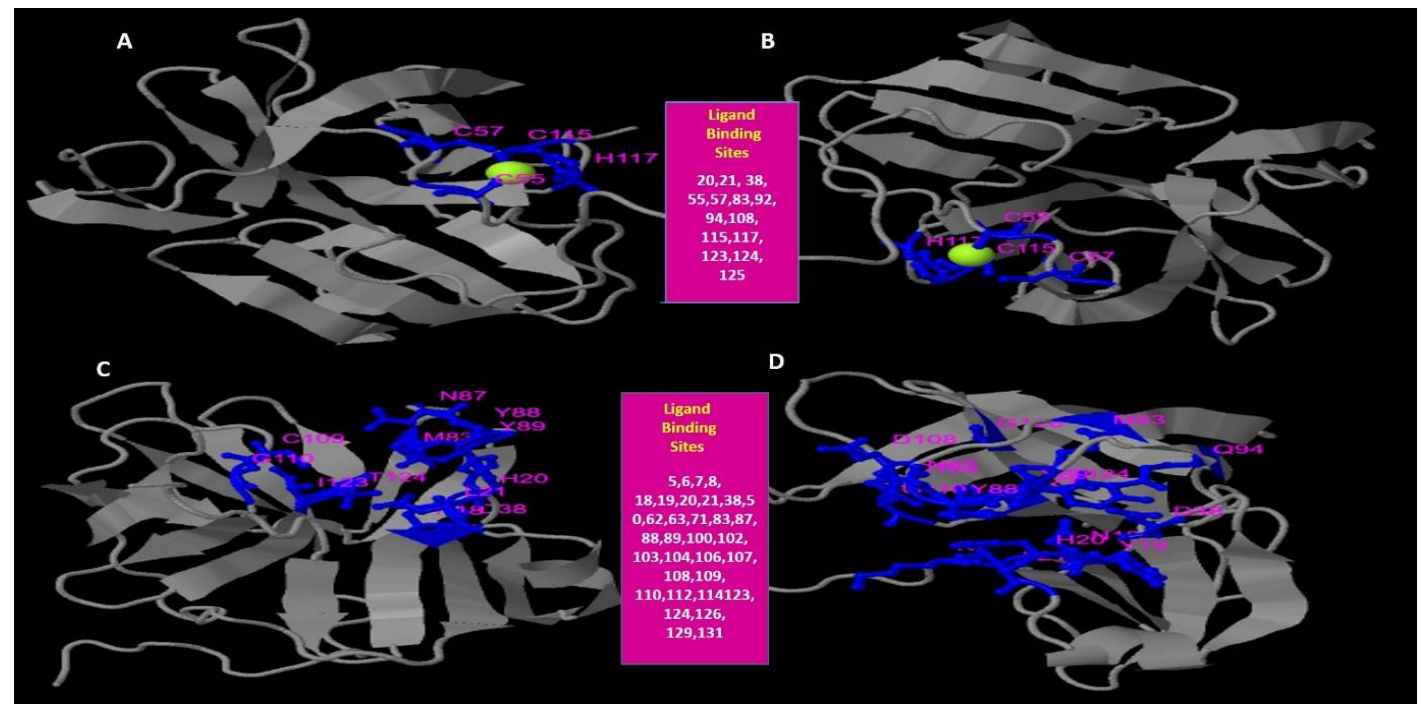

Figure 6: Ligand binding sites prediction through $\mathrm{COACH}$ server. $\mathrm{A}$ and $\mathrm{B}$ are the predictions from TM-SITE of 001 and 002 respectively while $C$ and $D$ are the results of ConCavity. Ligand binding sites are shown in purple colour

\section{Protein stability with mutations}

The protein stability change upon mutation was assessed by using I-Mutant 2.0, STRUM and EASE-MM web-servers. The results showed the effect of mutants on stability of protein with reliability index at $\mathrm{pH} 7.0$ and temperature $25^{\circ} \mathrm{C}$. Mutations (A12G, D36N, S134T, I136V) used in this study indicated a decrease in stability of the protein.

\section{Hydrophobicity}

Hydrophobic interactions play an important role in drug design involving biophysical events for instance protein-ligand or protein-protein binding.
Hydrophobic regions of the selected 001 and 002 proteins were determined with PEPTIDE 2 and TM-Finder. These software predicted the calculated hydrophobicity and helicity values, along with the predicted TM-segments of the proteins (Figure 8). In case of both proteins most residues were found to be hydrophobic (34.9\%) as compared to hydrophilic residues (11.4\%).

\section{DISCUSSION}

Poliovirus $2 \mathrm{~A}$ protease is involved in many physiological processes, making it an important target in the cysteine protease superfamily of proteins. However, the absence of a high resolution crystal structure of poliovirus $2 \mathrm{~A}$ 
protease is a limitation in understanding atomic details [10]. In this study, we took advantage of the PV2A ${ }^{\text {pro }}$ primary structural similarity to other viral proteases, specifically that from Coxsackievirus B4, and performed an in silico analysis of its structure. Poliovirus 2A protease sequences (001 and 002) were taken from our previous work where we have reported the mutations in this protein.

No previous studies have reported mutations detected in the poliovirus $2 \mathrm{~A}$ protease. In this study, we performed a computational analysis on these mutations to determine their impact on protease structure, function and stability. Intrinsic disordered region prediction results proved it to be suitable for drug binding sites, with a low number of residues lying in the disordered regions. Moreover, hydrophobicity results predicted that these proteases have more hydrophobic residues compared to hydrophilic residues. Important drug binding sites were predicted using the $\mathrm{COACH}$ server for these mutation containing proteases. These important residues include His20, Leu21, Asp38, Cys55, Cys57, Met83, Tyr88, Tyr89, Asp108, Cys109, Cyss115, His117, Ile123, Thr124, Ala125 and Gly126.
Previous studies have reported that His20, Cys109, Cys55, Cys57, Cys115 and His117 are involved in structural maintenance and catalytic activity of $2 A^{\text {pro }}$ [40]. Proteinase $2 A$ of human rhinovirus 2 (HRV2 2A) Cys residues and His114 are important residues involved in coordinating $\mathrm{Zn}^{+2}$. Moreover, Gly123, Gly124, Thr121 and Cys101 are suggested to form part of the construction of the substrate binding pocket and to offer a suitable setting for the active site of His18, Asp35 and Cys106 [41]

Previously reported mutations (A12G, D36N, $\mathrm{S} 134 \mathrm{~T}, \mathrm{I136 \textrm {V } )}$ in poliovirus $2 \mathrm{~A}$ protease were subjected to predict single nucleotide polymorphisms along with their impact on protease stability. All mutations result in a decrease of protein stability, thus destabilizing the protein structure. Defects in cis cleavage with the minute plaque phenotype have been observed in poliovirus point mutants (G60R, S66F, L39F, N18K, D135N, V119M, A22T, C55Y, and S134L) when the poliovirus $2 \mathrm{~A}$ gene was expressed in yeast [42]. Previously it was reported that single amino acid mutations at the His20 and Cysl09 residues led to complete loss of the proteolytic activity of 2Apro [43].

A

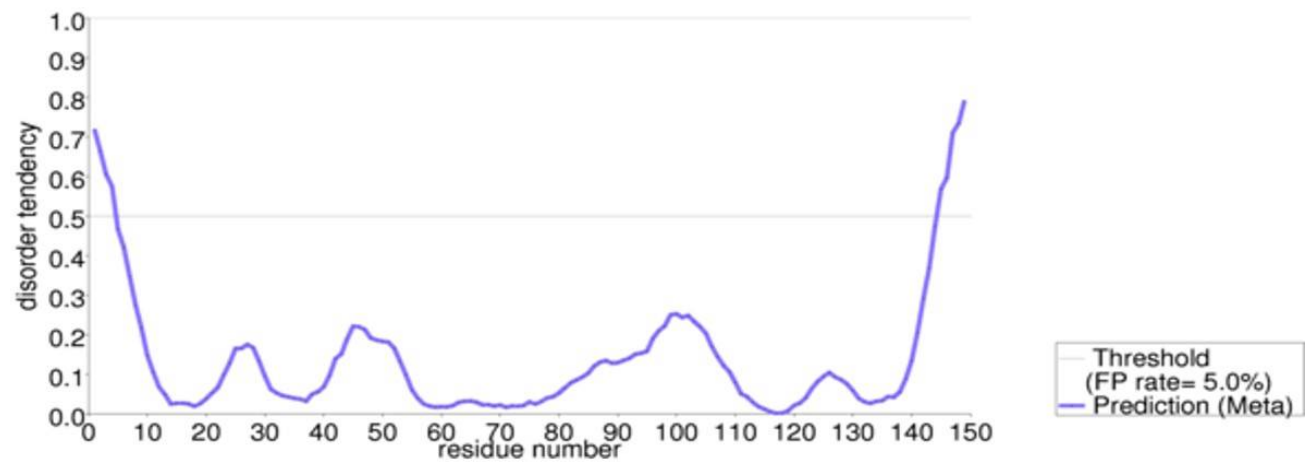

B
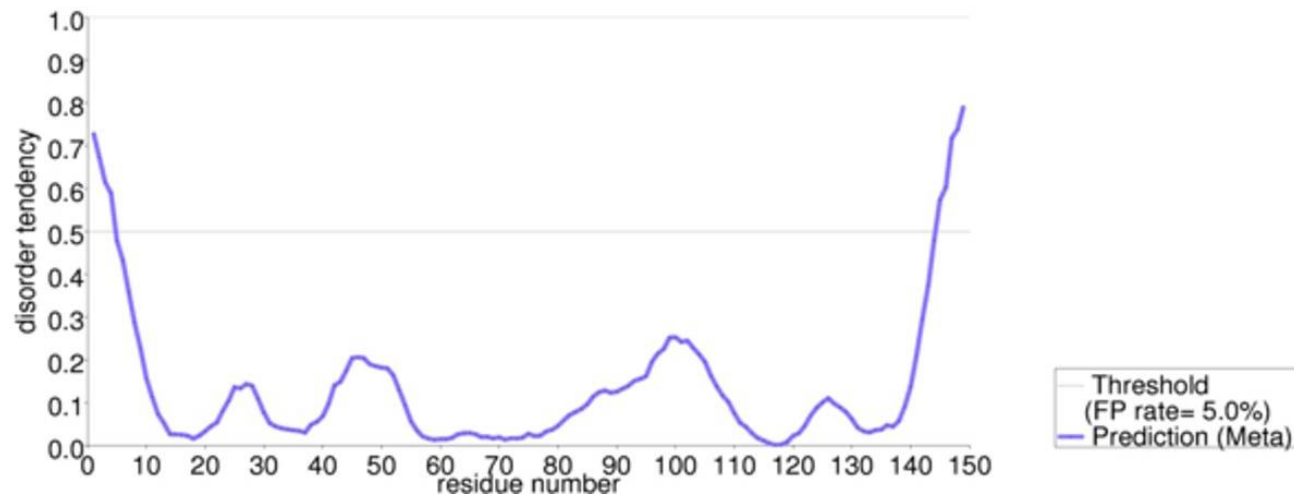

Figure 7: Intrinsic disorder regions of $001(\mathrm{~A})$ and 002 (B). Scores above the grey line are the disorder region, whereas scores below the grey line show degree of flexibility 

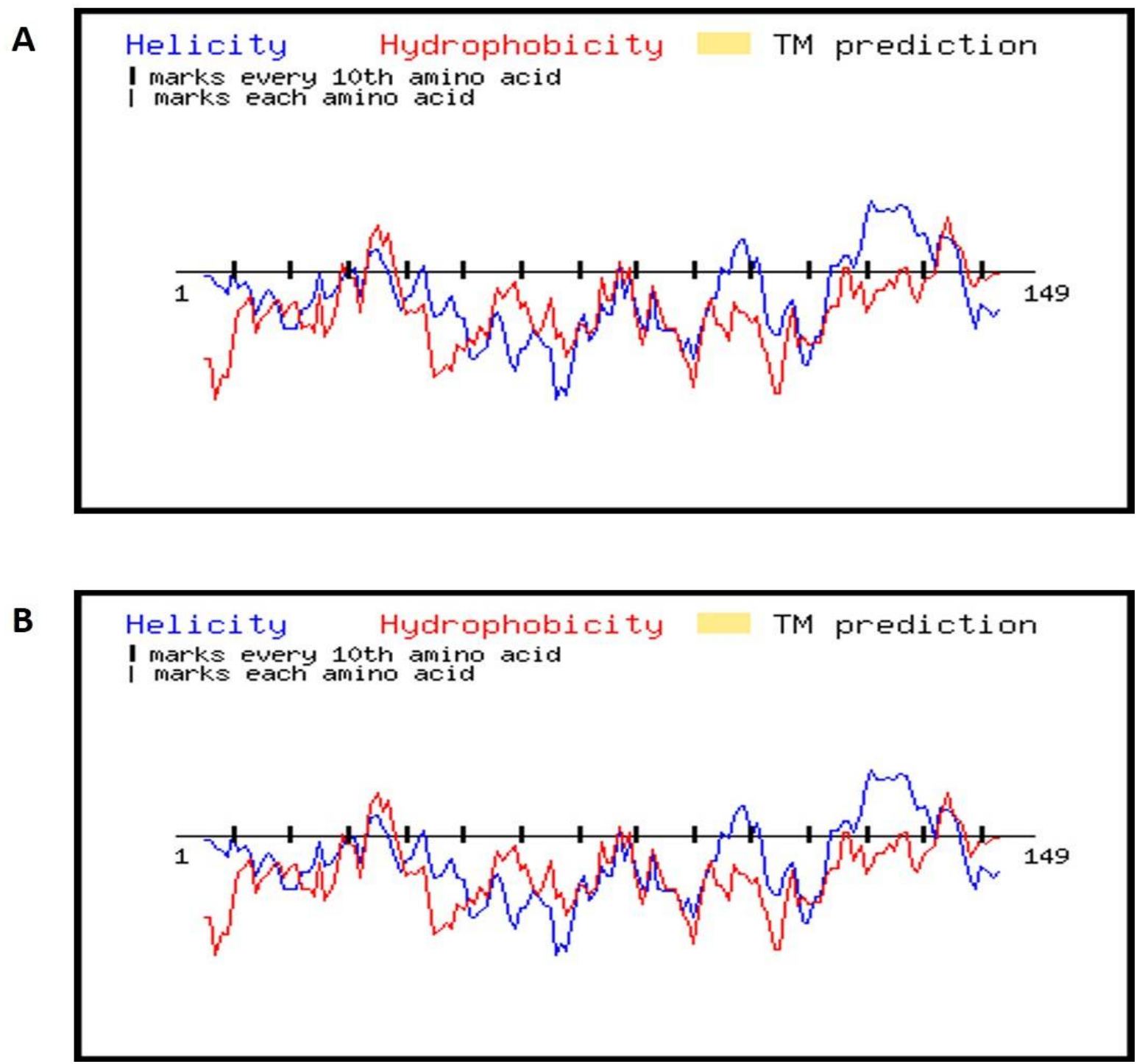

Figure 8: Hydrophobicity results of 001 (A) and 002 (B) model. Red amino acids are amino acids in a TM segment and Blue amino acids are charged amino acids in the TM segment

Important ligand-protein interactions have been identified following docking investigations in this study, providing potential candidates for drug targeting, the potency of which may be increased following relatively simple structural alterations.

\section{CONCLUSION}

A structure-function investigation of the poliovirus $2 \mathrm{~A}$ protease is presented in the study. By using the crystal structure of coxsackievirus B4 as a template, the three-dimensional structure of the poliovirus $2 \mathrm{~A}$ protease was predicted. I-TASSER and $\mathrm{COACH}$ results identified potential binding sites in the protein structure. Use of computational approaches to identify more potential drug binding sites in the conserved regions will lead to better drug design methods in the years ahead. This computational approach may be combined with experimental methods, thus contributing to functional interpretation of protein structures.

\section{DECLARATIONS}

\section{Acknowledgement}

The authors would like to acknowledge the guidance and examination committee members, including Nasar Virk, Hussnain Ahmed Janjua and Muhammad Arshad Malik, for their feedback on the work; Faryal Mehwish Awan and Aqsa Ikram for providing valuable suggestions; and National University of Sciences \& Technology (NUST) Islamabad for providing financial support.

\section{Conflict of interest}

The authors declare that no conflict of interest is 
associated with this study.

\section{Contribution of authors}

We declare that this work was done by the authors named in this article and all liabilities pertaining to claims relating to the content of this article will be borne by the authors.

\section{REFERENCES}

1. De Chassey B, Meyniel-Schicklin L, Aublin-Gex A, André $P$, Lotteau $V$ : New horizons for antiviral drug discovery from virus-host protein interaction networks. Curr Opin Virol 2012; 2: 606-613.

2. Landry $Y$, Gies JP. Drugs and their molecular targets: an updated overview. Fundam Clin Pharmacol 2008; 22: 18.

3. Eigen M. Viral quasispecies. Scientific AmericanAmerican edition. 1993; 269:42-49.

4. Holland J, Spindler K, Horodyski F, Grabau E, Nichol S, VandePol S. Rapid evolution of RNA genomes. Science 1982; 215: 1577-1585.

5. Domingo E, Menendez-Arias L, Holland JJ. RNA virus fitness. Rev Med Virol 1997; 7: 87-96.

6. Hogle J, Chow M, Filman D. Three-dimensional structure of poliovirus at $2.9 \mathrm{~A}$ resolution. Science 1985; 229: 1358-1365.

7. Kitamura N, Semler BL, Rothberg PG, Larsen GR, Adler CJ, Dorner AJ, Emini EA, Hanecak R, Lee JJ, van der Werf $S$, et al. Primary structure, gene organization and polypeptide expression of poliovirus RNA. Nature 1981; 291: 547-553.

8. Kuyumcu-Martinez NM, Joachims M, Lloyd RE. Efficient cleavage of ribosome-associated poly (A)-binding protein by enterovirus $3 C$ protease. J Virol 2002; 76 : 2062-7204.

9. Mueller S, Wimmer E, Cello J. Poliovirus and poliomyelitis: $A$ tale of guts, brains, and an accidental event. Virus Res 2005; 111: 175-193.

10. Petersen JF, Cherney MM, Liebig HD, Skern T, Kuechler $E$, James $M N$. The structure of the $2 A$ proteinase from a common cold virus: a proteinase responsible for the shut-off of host-cell protein synthesis. EMBO J 1999; 18 : 5463-5475.

11. Crowder S, Kirkegaard K. Trans-dominant inhibition of RNA viral replication can slow growth of drug-resistant viruses. Nat Genet 2005; 37: 665-666.

12. National Research Council. Exploring the role of antiviral drugs in the eradication of polio: Workshop report. National Academies Press, 2006.

13. Younus A, Bhatti MF, Ahmad T, Virk N, Arshad M, Janjua $H A$. Protein structure modelling, ligand docking and active site analysis of mutated poliovirus $2 A$ protease gene isolated from the blood of Pakistani polio infected patients. Int J Adv Sci Engine Technol 2016; 4.

14. Biasini M, Bienert S, Waterhouse A, Arnold K, Studer G, Schmidt T, Kiefer F, Cassarino TG, Bertoni M, Bordoli L, et al. SWISS-MODEL: modelling protein tertiary and quaternary structure using evolutionary information. Nucleic Acids Res 2014; 42: W252-W258.

15. Ramachandran GN, Ramakrishnan C, Sasisekharan V. Stereochemistry of polypeptide chain configurations. $J$ Mol Biol 1963; 7: 95-99.

16. Benkert $P$, Künzli $M$, Schwede T. QMEAN server for protein model quality estimation. Nucleic Acids Res 2009; 37: W510-W514.

17. Wiederstein M, Sippl MJ. ProSA-web: interactive web service for the recognition of errors in three-dimensional structures of proteins. Nucleic Acids Res 2007; 35: W407-W410.

18. Willard L, Ranjan A, Zhang $H$, Monzavi $H$, Boyko RF, Sykes BD, Wishart DS. VADAR: a web server for quantitative evaluation of protein structure quality. Nucleic Acids Res 2003; 31: 3316-3319.

19. Zhang Y. I-TASSER server for protein $3 D$ structure prediction. BMC Bioinformatics 2008; 9: 40.

20. Roy A, Yang J, Zhang Y. COFACTOR: an accurate comparative algorithm for structure-based protein function annotation. Nucleic Acids Res 2012; 40: W471W477.

21. Bava KA, Gromiha MM, Uedaira $H$, Kitajima K, Sarai A. ProTherm, version 4.0: thermodynamic database for proteins and mutants. Nucleic Acids Res 2004; 32: D120-D121.

22. Capriotti E, Fariselli P, Casadio R. A neural-networkbased method for predicting protein stability changes upon single point mutations. Bioinformatics 2004; 20 : 163-168.

23. Quan L, Lv Q, Zhang Y. STRUM: structure-based prediction of protein stability changes upon single-point mutation. Bioinformatics 2016; 32: 2936-2946.

24. Folkman L, Stantic B, Sattar A, Zhou Y. EASE-MM: Sequence-based prediction of mutation-induced stability changes with feature-based multiple models. $\mathrm{J} \mathrm{Mol} \mathrm{Biol}$ 2016; 428: 1394-1405.

25. Ishida T, Kinoshita K. Prediction of disordered regions in proteins based on the meta approach. Bioinformatics 2008; 24: 1344-1348.

26. Linding $R$, Jensen LJ, Diella $F$, Bork $P$, Gibson $T J$, Russell RB. Protein disorder prediction: implications for structural proteomics. Structure 2003; 11: 1453-1459.

27. Buchan DW, Minneci F, Nugent TC, Bryson $K$, Jones DT. Scalable web services for the PSIPRED Protein Analysis Workbench. Nucleic Acids Res 2013; 41: W349-W357.

28. Deng X, Eickholt J, Cheng J. PreDisorder: ab initio sequence-based prediction of protein disordered regions. BMC Bioinformatics 2009; 10: 1 .

29. Deber CM, Wang C, Liu LP, Prior AS, Agrawal S, Muskat BL, Cuticchia AJ. TM Finder: a prediction program for transmembrane protein segments using a combination of hydrophobicity and nonpolar phase helicity scales. Protein Sci 2001; 10: 212-219.

30. Yang J, Roy A, Zhang Y. Protein-ligand binding site recognition using complementary binding-specific 
substructure comparison and sequence profile alignment. Bioinformatics 2013; 29: 2588-2595.

31. Wang W, Malcolm BA. Two-stage polymerase chain reaction protocol allowing introduction of multiple mutations, deletions, and insertions, using QuikChange TM site-directed mutagenesis. In Vitro Mutagenesis Protocols 2002:37-43.

32. Capra JA, Laskowski RA, Thornton JM, Singh M, Funkhouser TA. Predicting protein ligand binding sites by combining evolutionary sequence conservation and 3D structure. PLoS Comp Biol 2009; 5: e1000585.

33. Skolnick J, Brylinski M. FINDSITE: a combined evolution/structure-based approach to protein function prediction. Brief Bioinform 2009; 10: 378-391.

34. Barh D, Tiwari S, Jain N, Ali A, Santos AR, Misra AN, Azevedo V, Kumar A. In silico subtractive genomics for target identification in human bacterial pathogens. Drug Dev Res 2011; 72: 162-177.

35. Yu CS, Chen YC, Lu CH, Hwang JK. Prediction of protein subcellular localization. Proteins: Structure, Function, and Bioinformatics 2006; 64: 643-651.

36. Shen HB, Chou KC. Virus-mPLoc: a fusion classifier for viral protein subcellular location prediction by incorporating multiple sites. J Biomol Struct Dyn 2010; 28: 175-186.
37. Bernsel A, Viklund $H$, Hennerdal A, Elofsson $A$. TOPCONS: consensus prediction of membrane protein topology. Nucleic Acids Res 2009; 37: W465-W468.

38. Krogh A, Larsson B, Von Heijne G, Sonnhammer EL. Predicting transmembrane protein topology with a hidden Markov model: application to complete genomes. J Mol Biol 2001; 305: 567-580.

39. Benkert P, Biasini M, Schwede T. Toward the estimation of the absolute quality of individual protein structure models. Bioinformatics 2010; 27: 343-350.

40. Yu SF, Lloyd RE. Characterization of the roles of conserved cysteine and histidine residues in poliovirus $2 A$ protease. Virology 1992; 186: 725-735.

41. Sommergruber W, Seipelt J, Fessl F, Skern T, Liebig HD, Casari G. Mutational Analyses Support a Model for the HRV2 2 A Proteinase. Virology 1997; 234: 203-214.

42. Barco A, Ventoso I, Carrasco L. 1997. The yeast Saccharomyces cerevisiae as a genetic system for obtaining variants of poliovirus protease $2 A$. J Biol Chem 1997; 272:12683-12691.

43. Shuyuarn FY, Lloyd RE. Identification of essential amino acid residues in the functional activity of poliovirus $2 A$ protease. Virology 1991; 182: 615-625. 